\title{
SLC39A4 as a Novel Prognosis Marker Promotes Tumor Progression in Esophageal Squamous Cell Carcinoma
}

This article was published in the following Dove Press journal: OncoTargets and Therapy

\author{
Chenmei Xia' \\ Xia Chen' \\ Jun $\mathrm{Li}^{2}$ \\ Peng Chen ${ }^{3}$ \\ 'Department of Gastroenterology, The \\ First People's Hospital of Wenling, \\ Wenling 317500, People's Republic of \\ China; ${ }^{2}$ Department of Joint Surgery, The \\ First People's Hospital of Wenling, \\ Wenling 317500, People's Republic of \\ China; ${ }^{3}$ Department of Gastrointestinal \\ Surgery, The First People's Hospital of \\ Wenling, Wenling 317500, People's \\ Republic of China
}

Correspondence: Peng Chen Department of Gastrointestinal Surgery, The First People's Hospital of Wenling, Wenling 317500, Zhejiang Province, People's Republic of China Tel +86-0576-89668222

Email drchen65@I63.com
Background: Solute carrier family 39 member 4 (SLC39A4) has been reported to play an oncogenic role in several cancers. However, the role of SLC39A4 in esophageal squamous cell carcinoma (ESCC) remains unclear. In this study, we aimed to explore the clinical significance and function of SLC39A4 in ESCC.

Methods: The Cancer Genome Atlas and Gene Expression Omnibus databases were analyzed to assess the level of SLC39A4 in ESCC. The expression level of SLC39A4 was measured by RT-qPCR and immunohistochemistry in a cohort of 73 patients aged 45-65 years with ESCC. Kaplan-Meier analysis was used to identify the correlation between SLC39A4 and the prognosis of ESCC patients. In vitro experiments were conducted to explore the biological function of SLC39A4 in ESCC cell line TE-1 and TE-10.

Results: The mRNA level of SLC39A4 was significantly enhanced in ESCC specimens, which was in line with the outcome of online databases analysis. Moreover, the aberrant expression of SLC39A4 was positively correlated with clinical stage, T categories and lymph node metastasis. Kaplan-Meier analysis indicated that elevated SLC39A4 expression predicted poor prognosis of patients with ESCC. Furthermore, the in vitro experiments showed that SLC39A4 knockdown not only impaired the proliferation and motility capacities of ESCC cells but also enhanced the sensitivity to cisplatin treatment.

Conclusion: Our findings suggest that SLC39A4 could serve as a novel prognosis biomarker to promote ESCC progression; however, the mechanism of SLC39A4 in ESCC remains to be further explored.

Keywords: esophageal squamous cell carcinoma, SLC39A4, proliferation, metastasis, chemosensitivity, epithelial-mesenchymal transition

\section{Introduction}

As one of the most aggressive carcinomas, esophageal cancer (EC) ranks the 8th most common and the 6th most lethal cancer around the world. ${ }^{1}$ Esophageal squamous cell carcinoma (ESCC), as a dominant histologic form of EC, accounts for $90 \%$ of EC worldwide. ${ }^{2}$ Although developed systemic therapies have improved outcomes, the 5-year overall survival rate of patients with ESCC remains at $15-25 \%,{ }^{3}$ mainly caused by local recurrence and distant metastasis. ${ }^{4}$ Therefore, there is an urgent need to seek potential targets for clinical diagnosis and treatment to improve prognosis of ESCC patients.

The solute carrier (SLC) group of membrane transport proteins are involved in various physiological processes. ${ }^{5,6}$ As one of membrane transport proteins of SLC 
family, Zinc transporters consist of solute carrier family 30 (SLC30) and solute carrier family 39 (SLC39). ${ }^{7,8}$ SLC39, also known as Zrt- and Irt-like protein (ZIP), could play a crucial role in human diseases including malignant tumor by controlling the influx of zinc. ${ }^{9-11}$ Cui et al demonstrated that suppression of SLC39A6 could abrogate proliferation and invasion and stimulate apoptosis in ESCC. ${ }^{12}$ Similarly, SLC39A7 improved proliferation and survival of colorectal cancer cells. ${ }^{13}$ In metastatic breast cancer, SLC39A10 was positively correlated with lymph node metastasis and participated in the cell migratory activity. ${ }^{14}$ Therefore, the members of SLC39 may be utilized as diagnostic and therapeutic targets for human cancers.

SLC39A4, also known as ZIP4, plays a crucial role in maintaining physiologic concentrations of intracellular zinc ion to affect diverse biological processes. ${ }^{15-17}$ In pancreatic cancer, SLC39A4 promoted cell proliferation, resistance to apoptosis and cachexia. ${ }^{18-20}$ Besides, suppression of SLC39A4 impeded tumor metastasis and chemoresistance in non-small cell lung cancer. ${ }^{21}$ Moreover, SLC39A4 was verified to be a novel marker to predict prognosis in multiple cancers. ${ }^{21,22}$ However, the role of SCL39A4 in ESCC is still unclear. In this study, we aimed to clarify the clinical significance of SLC39A4 in ESCC, and further assess the impact of SLC39A4 on cell proliferation, motility and chemosensitivity of ESCC cells.

\section{Materials and Methods}

\section{Cell Lines and Clinical Tissue Samples}

The human ESCC cell lines TE-1 and TE-10 were purchased from Shanghai Institutes for Biological Sciences. Both cells were cultured in RPMI 1640 supplemented with $10 \% \mathrm{FBS}, 100 \mathrm{U} / \mathrm{mL}$ penicillin and $100 \mathrm{mg} / \mathrm{mL}$ streptomycin. The two cell lines were authenticated using Short Tandem Repeat (STR) analysis.

ESCC samples $(\mathrm{N}=73)$ and normal esophageal tissues $(\mathrm{N}=21)$ were collected by surgical resection for RNA extraction or paraffin embedding. The malignant tissues were verified by two pathologists independently. For RNA extraction, the specimens were snap frozen in liquid nitrogen. All patients whose tissues were used in this research had provided written informed consent, in accordance with the Declaration of Helsinki. This study was approved by the ethical committee of the First People's Hospital of Wenling, and the reference number of the approval was 2018-05-10.

\section{Bioinformatics Analysis}

The online databases including The Cancer Genome Atlas (TCGA), Genotype-Tissue Expression (GTEx) and Gene Expression Omnibus (GEO) were used to analyze the expression of SLC39A4 in ESCC and normal specimens.

\section{Antibodies and Reagents}

The following antibodies are used for immunoblotting in this study: SLC39A4, Proteintech (Rosemont, IL, USA), 206251-AP, 1:1000; Cyclin D1, Cell Signaling Technology (Danvers, MA, USA), \#55506, 1:1000; E-cadherin, Cell Signaling Technology, \#3195, 1:1000; N-cadherin, Cell Signaling Technology, \#13116, 1:1000; Vimentin, Cell Signaling Technology, \#5741, 1:1000; Snail, Cell Signaling Technology, \#3879, 1:1000; GAPDH, Santa Cruz (Dallas, Texas, USA), sc-365062, 1:5000. Cisplatin and N,N,N',N'tetrakis(2-pyridylmethyl)ethylenediamine (TPEN) were obtained from Sigma-Aldrich (St. Louis, MO, USA).

\section{RNA Extraction and RT-qPCR}

RNA in clinical tissue samples (21 cases of ESCC specimens and 21 cases of normal esophageal tissues) and ESCC cells were extracted using TRIzol reagent (Thermo Fisher Scientific, Waltham, MA, USA) according to the manufacturer's instruction and then were reversely transcribed to cDNA using FastQuant RT Super Mix (TIANGEN Biotech, Beijing, China). The mRNA of SLC39A4 was detected on Applied Biosystems 7500 Real-Time PCR System (Thermo Fisher Scientific) by using SYBR ${ }^{\circledR}$ Premix Ex Taq $^{\text {TM }}$ II (TaKaRa, Dalian, China). GAPDH was used as an internal control. The primer pairs of target mRNAs were as follows: SLC39A4, F: 5'- CGAGGTCCCTATGACGCTG-3' and R: 5'-CACTCAGGCATACCGTGTCC-3', GAPDH, F: 5'TGCACCACCAACTGCTTAGC-3' and R: 5'GGCATGGACTGTGGTCATGAG-3'.

\section{Immunohistochemistry}

Paraffin-embedded ESCC tissues $(\mathrm{N}=73)$ were deparaffinized and rehydrated. After heat-induced epitope antigen retrieval with citrate buffer in the microwave, the specimens were incubated with primary antibody against SLC39A4 (1:100, Proteintech) overnight at $4^{\circ} \mathrm{C}$, followed by incubation with secondary antibody for $30 \mathrm{~min}$ at room temperature. Slides were developed with a DAB Chromogen kit (ZSGB-BIO, Beijing, China) and counterstained with hematoxylin. The expression of SLC39A4 
was measured by assessing both the staining intensity (a: 0 , negative; 1 , weak positive; 2 , moderate positive; 3 , strong positive) and the percentage of positively stained cells (b: $0,0-5 \%$ positive cells; $1,6-25 \%$ positive cells; 2 , $26-50 \%$ positive cells and $3,51-100 \%$ positive cells). The score of each slide was determined by the product of a and b. The scores of $\leq 3$ were regarded as low expression, while the ones of $>3$ indicated high expression.

\section{Transfection}

The plasmid overexpressing SLC39A4 (pEnter-SLC39A4) was purchased from Vigene Biosciences (Shandong, China), and SLC39A4 siRNAs and negative control were obtained from RIBOBIO (Guangzhou, China). The plasmid or siRNAs was transfected into ESCC cells using Lipofectamine 3000 (Thermo Fisher Scientific) according to the manufacturer's instruction. After 48 hours, the mRNA and protein expression of SLC39A4 were measured and functional assays were conducted.

\section{Immunoblotting}

Cells were lysed by using RIPA lysis buffer with protease/ phosphatase inhibitor cocktail (Roche, Basel, Switzerland). After quantification and denaturation, proteins $(30 \mu \mathrm{g})$ were separated with SDS-PAGE and transferred to PVDF membranes (Thermo Fisher Scientific). The membranes were incubated with primary antibodies overnight at $4^{\circ} \mathrm{C}$. The protein expressions were then detected using enhanced Chemiluminescence (Solarbio, Beijing, China). ImageJ software was used to quantify the protein expression.

\section{CCK-8 Assay}

Cell viability was detected using CCK-8 kit (Solarbio). TE-1 (2000 cells per well) or TE-10 cells (2000 cells per well) were seeded in 96-well plates and cultured for 4 consecutive days. When the cells were incubated with CCK-8 for 2 hours, OD values at $450 \mathrm{~nm}$ were measured.

For testing the response of ESCC cells to CDDP, the ESCC cells (5000 cells per well in 96-well plates) were exposed to a range of concentrations of drugs respectively $(0,1,2,5,10$, $50,100,500,1000 \mu \mathrm{M})$. The OD values were obtained 24 hours after the drugs treatment and the half maximal inhibitory concentration $\left(\mathrm{IC}_{50}\right)$ to $\mathrm{CDDP}$ were determined by using GraphPad Prism 6.0 (La Jolla, CA, USA). When the concentration of cisplatin is $0 \mu \mathrm{M}$, the inhibition rate is $0 \%$; once drug dosage reaches to $1000 \mu \mathrm{M}$, all cells die and the inhibition rate is $100 \%$. After transformation and normalization, nonlinear regression is performed for $\mathrm{IC}_{50}$ calculation according to the instruction of GraphPad (https://www.graphpad.com/support/ faq/how-to-determine-an-icsub50sub/).

\section{Clonogenicity Assay}

ESCC cells (500 cells/well) were cultured in complete medium in a 6-well plate for 2 weeks. Then the cells were fixed and stained with $0.1 \%$ crystal violet. Colonies with more than 50 cells were counted.

\section{Transwell Assay}

For in vitro invasion or migration assays, TE-1 or TE-10 cells $(50,000$ cells/well) were plated into Transwell inserts (Corning, NY, USA) pre-coated with Matrigel (BD Biosciences, Franklin Lakes, NJ, USA) or not. The lower chambers were filled with $600 \mu \mathrm{L}$ RPMI 1640 medium supplemented with 20\% FBS. After incubation for 24 hours, the penetrated cells were washed, fixed and then stained with $0.1 \%$ crystal violet.

\section{Flow Cytometry}

To determine cell-cycle distribution, cells transfected with siNC or siSLC39A4 were fixed in $75 \%$ ethanol, washed twice with PBS, and incubated in $500 \mu \mathrm{L}$ PBS containing $10 \mu \mathrm{L}$ PI $(1 \mathrm{mg} / \mathrm{mL})$ and $0.5 \mu \mathrm{L}$ RNase $(10 \mathrm{mg} / \mathrm{mL})$ at $37^{\circ}$ $\mathrm{C}$ for 30 minutes in the dark. Cell cycle was then assessed using flow cytometry (FACS Canto ${ }^{\mathrm{TM}}$ II, BD).

For apoptosis analysis, the siSLC39A4-transfected ESCC cells treated with CDDP for $24 \mathrm{hr}$, were collected and stained using Annexin V-FITC/PI Apoptosis detection Kit II (BD) for 15 minutes according to the recommendation of manufacturer. Then, the apoptosis rate was detected on FACS Canto ${ }^{\mathrm{TM}}$ II (BD).

\section{Statistical Analysis}

Statistical analyses were conducted using GraphPad Prism 6.0 software. Data were presented as mean \pm S.D. Comparisons between two groups were carried out using Student's $t$-tests. A value of $P<0.05$ was considered statistically significant.

\section{Results}

SLC39A4 Is Aberrantly Expressed in ESCC and Indicates Unfavorable Prognosis.

Firstly, we assessed the expression of SLC39A4 in TCGA database by using GEPIA online software (gepia.cancer-pku. cn) and found that SLC39A4 was significantly increased in various cancers including esophageal cancer (Figure 1A). In 
the meanwhile, mining five accessible datasets of gene expression profiling (GSE17351, GSE20347, GSE23400, GSE38129 and GSE100942) in GEO also confirmed that SLC39A4 was elevated in ESCC tissues (Figure 1B). The qPCR results showed that the mRNA of SLC39A4 was enhanced in ESCC specimens relative to normal esophageal tissues in our cohort ( $\mathrm{N}=21$, Figure $1 \mathrm{C})$, which was in accordance with the results of online databases analysis above. Then, immunohistochemistry assay was performed to determine SLC39A4 protein expression in ESCC (Figure 1D). The
A

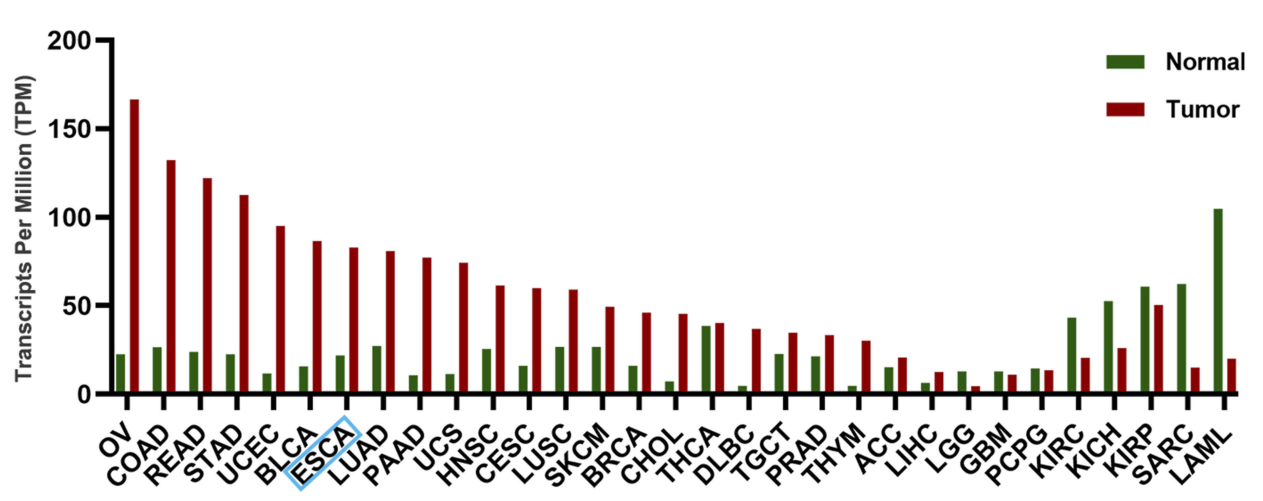

B
GSE20347

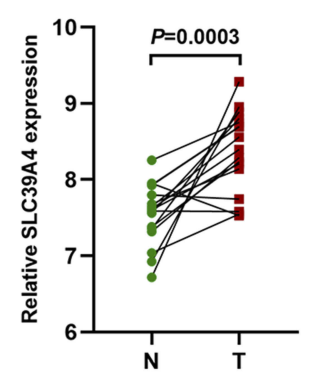

D

C
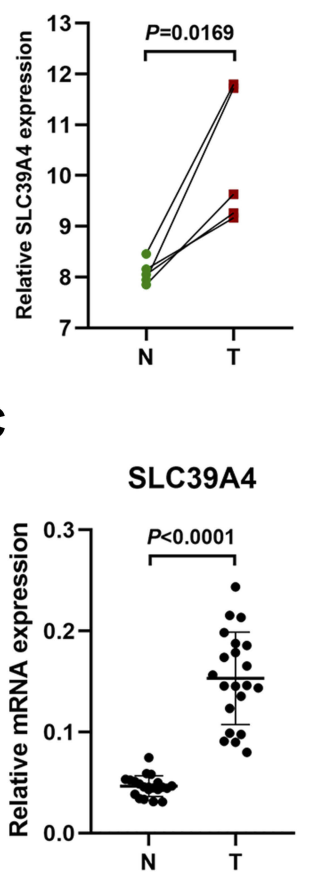

GSE23400

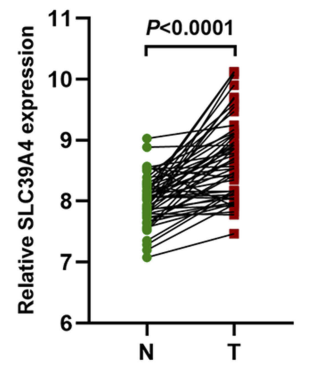

GSE38129

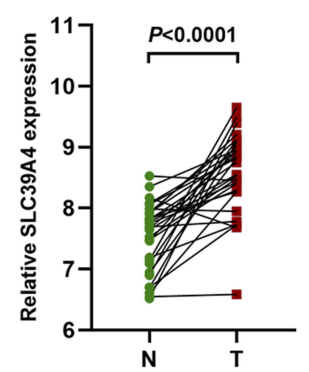

SLC39A4

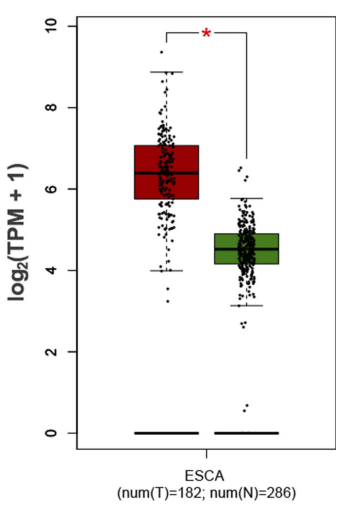

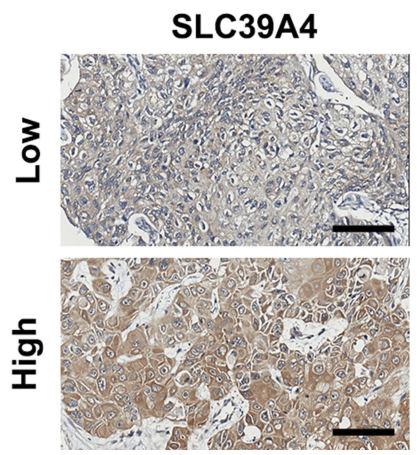

E

SLC39A4

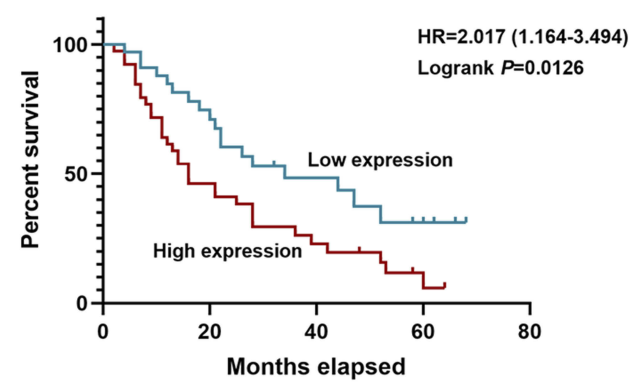

Figure I Enhanced expression of SLC39A4 in ESCC tissues indicates poor prognosis in ESCC patients. (A) The level of SLC39A4 across various cancers including esophageal cancer compared to normal tissues. TCGA and GTEx datasets were used to analyze SLC39A4 expression in both tumor and normal specimens. Data, mean \pm SD, $* P<0.05$. (B) The analysis of GEO database (GSEI735I, GSE20347, GSE23400, GSE38129 and GSE 100942) indicated that the mRNA level of SLC39A4 was significantly elevated in ESCC tissues. (C) The qPCR results showed that the mRNA level of SLC39A4 in ESCC tissues $(N=21)$ was higher relative to the normal ones $(N=21)$. Data, mean \pm SD. (D) Representative photographs of IHC results of SLC39A4 in ESCC. Scale bar, $100 \mu \mathrm{m}$. (E) Increased SLC39A4 expression indicates poor overall survival in ESCC patients.

Abbreviations: OV, ovarian serous cystadenocarcinoma; COAD, colon adenocarcinoma; READ, rectum adenocarcinoma; STAD, stomach adenocarcinoma; UCEC, uterine corpus endometrial carcinoma; BLCA, bladder urothelial carcinoma; ESCA, esophageal cancer; LUAD, lung adenocarcinoma; PAAD, lung adenocarcinoma; UCS, uterine carcinosarcoma; HNSC, head and neck squamous cell carcinoma; CESC, cervical squamous cell carcinoma and endocervical adenocarcinoma; LUSC, lung squamous cell carcinoma; SKCM, skin cutaneous melanoma; BRCA, breast invasive carcinoma; CHOL, cholangio carcinoma; THCA, thyroid carcinoma; DLBC, lymphoid neoplasm diffuse large B-cell lymphoma; TGCT, testicular germ cell tumors; PRAD, prostate adenocarcinoma; THYM, thymoma; ACC, adrenocortical carcinoma; LIHC, liver hepatocellular carcinoma; LGG, brain lower grade glioma; GBM, glioblastoma multiforme; PCPG, pheochromocytoma and paraganglioma; KIRC, kidney renal clear cell carcinoma; KIRP, kidney renal papillary cell carcinoma; SARC, sarcoma; LAML, acute myeloid leukemia; HR, hazard ratio; T, tumor; N, normal tissue 
expression of SLC39A4 was positively correlated with clinical stage, T categories and lymph node metastasis in ESCC (Table 1). Kaplan-Meier survival analysis revealed that aberrant expression of SLC39A4 predicted poor prognosis of patients with $\operatorname{ESCC}(\mathrm{HR}=2.017, P=0.0126$, Figure 1E). Together, these results suggest that SCL39A4 probably plays a critical role in ESCC progression and even could be a potential prognostic marker in ESCC.

\section{SLC39A4 Facilitates the Proliferation of ESCC Cells in vitro}

Next, loss-of-function and gain-of-function assays were performed. TE-1 and TE-10 cells were transfected with specific siRNAs targeting SLC39A4. The knockdown efficacy was evaluated using qPCR and immunoblotting assays (Figure 2A and B). We then found that the growth rate was retarded in SLC39A4-deficient ESCC cells compared to the control cells transfected with scramble siRNA (Figure 2C). Besides, knockdown of SLC39A4 significantly reduced both the size and number of colonies in

Table I SLC39A4 Expression and Clinicopathological Characteristics of ESCC

\begin{tabular}{|c|c|c|c|c|}
\hline \multirow[t]{2}{*}{ Characteristics } & \multicolumn{2}{|c|}{ SLC39A4 Expression } & \multirow[t]{2}{*}{ Total } & \multirow[t]{2}{*}{$P$ value } \\
\hline & Low & High & & \\
\hline Overall & $34(46.6 \%)$ & 39 (53.4\%) & 73 & \\
\hline $\begin{array}{l}\text { Sex } \\
\qquad \text { Male } \\
\text { Female }\end{array}$ & $\begin{array}{l}26(46.4 \%) \\
8(47.1 \%)\end{array}$ & $\begin{array}{l}30(53.6 \%) \\
9(52.9 \%)\end{array}$ & $\begin{array}{l}56 \\
17\end{array}$ & 0.964 \\
\hline $\begin{array}{l}\text { Age } \\
\qquad \begin{array}{l}<0 \\
\geq 60\end{array}\end{array}$ & $\begin{array}{l}22(5 \mid .2 \%) \\
12(40.0 \%)\end{array}$ & $\begin{array}{l}2 \mid(48.8 \%) \\
\text { I 8(60.0\%) }\end{array}$ & $\begin{array}{l}43 \\
30\end{array}$ & 0.347 \\
\hline $\begin{array}{l}\text { Clinical stage } \\
\text { I-II } \\
\text { III-IV }\end{array}$ & $\begin{array}{l}23(59.0 \%) \\
\text { I I (32.4\%) }\end{array}$ & $\begin{array}{l}16(41.0 \%) \\
23(67.6 \%)\end{array}$ & $\begin{array}{l}39 \\
34\end{array}$ & $0.023^{*}$ \\
\hline $\begin{array}{l}\text { T categories } \\
\text { TI-T2 } \\
\text { T3-T4 }\end{array}$ & $\begin{array}{l}24(57.1 \%) \\
10(32.3 \%)\end{array}$ & $\begin{array}{l}\text { I8 }(42.9 \%) \\
2 \text { I }(67.7 \%)\end{array}$ & $\begin{array}{l}42 \\
31\end{array}$ & $0.035^{*}$ \\
\hline $\begin{array}{l}\mathrm{N} \text { metastasis } \\
\mathrm{N} 0 \\
\mathrm{~N} 1+\mathrm{N} 2+\mathrm{N} 3\end{array}$ & $\begin{array}{l}27(56.3 \%) \\
7(28.0 \%)\end{array}$ & $\begin{array}{l}2 \mid(43.8 \%) \\
18(72.0 \%)\end{array}$ & $\begin{array}{l}48 \\
25\end{array}$ & $0.022^{*}$ \\
\hline $\begin{array}{l}\text { Differentiation } \\
\text { Well } \\
\text { Moderately } \\
\text { Poorly }\end{array}$ & $\begin{array}{l}10(40.0 \%) \\
16(51.6 \%) \\
8(47.1 \%)\end{array}$ & $\begin{array}{l}\text { I5 (60.0\%) } \\
\text { I5 (48.4\%) } \\
9(52.9 \%)\end{array}$ & $\begin{array}{l}25 \\
31 \\
17\end{array}$ & 0.687 \\
\hline
\end{tabular}

Notes: The data were analyzed by Pearson chi-square test. $P$ values with significance were shown as an asterisk. $* P<0.05$. LN, lymph node.
TE-1 and TE-10 cells (Figure 2D). On the contrary, when SLC39A4 expression was elevated (Figure 2E), the colony formation capacity of ESCC cells was significantly enhanced (Figure 2F). As shown in Figure 2G and $\mathrm{H}$, we observed that cell cycle was arrested at G1 phase in both TE-1 and TE-10 cells transfected with siRNAs against SLC39A4. Meanwhile, the expression of Cyclin D1, a cell cycle-related molecule, was remarkably reduced (Figure 2I). Collectively, these data demonstrate that SLC39A4 improves the growth and proliferation of ESCC cells.

\section{SLC39A4 Enhances Motility Capacity of ESCC Cells in vitro}

In view of the positive correlation between SLC39A4 expression and lymph node metastasis, the effect of SLC39A4 on motility ability of ESCC cells in vitro was detected. Upon delivery of SLC39A4 siRNAs, the migration and invasion capacities of TE-1 cells were significantly impeded (Figure 3A, upper panels). Similar inhibitory effect was also observed in TE-10 cells with deficient SLC39A4 (Figure 3A, lower panels). As shown in Figure 3B, elevated SLC39A4 significantly promoted migration and invasion of ESCC cells in vitro. Epithelialmesenchymal transition (EMT) is one of major contributors to tumor distant metastasis. Therefore, the expressions of EMT markers such as E-cadherin and Snail were measured in the ESCC cells transfected with siRNA against SLC39A4. The results of immunoblotting showed that SLC39A4 knockdown led to an increase of E-cadherin, while the levels of N-cadherin, Vimentin and Snail were reduced (Figure 3C), suggesting that suppression of SLC39A4 reversed EMT process. SLC39A4 is critical for maintaining intracellular zinc concentration, therefore, the effect of $\mathrm{Zn}^{2+}$ depletion on EMT process was determined by using a zinc chelator TPEN. As shown in Figure 3D, the losses of N-cadherin, Vimentin and Snail were observed in TPEN-treated cells, while E-cadherin expression was elevated, similar to the effect of siSLC39A4 on EMT markers. Taken together, the above results demonstrate that SLC39A4 could promote cell motility by affecting EMT process in ESCC in vitro.

\section{SLC39A4 Strengthens Resistance of ESCC Cells to CDDP in vitro}

In addition to the malignant proliferation and distant metastasis, intrinsic or acquired resistance to chemotherapy is 
A

C
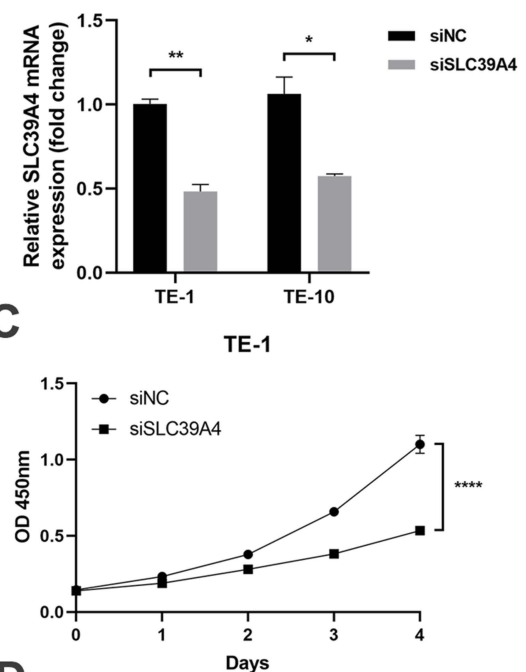

B

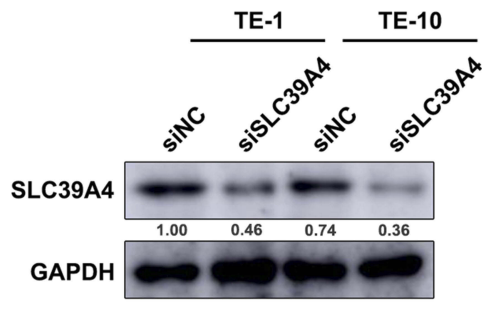

TE-10

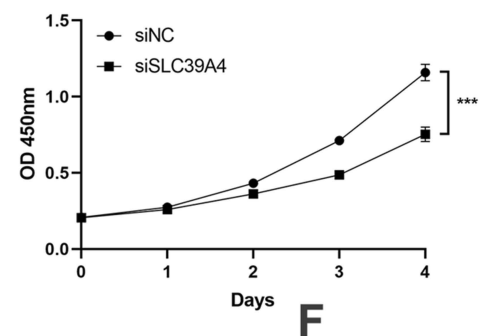

E
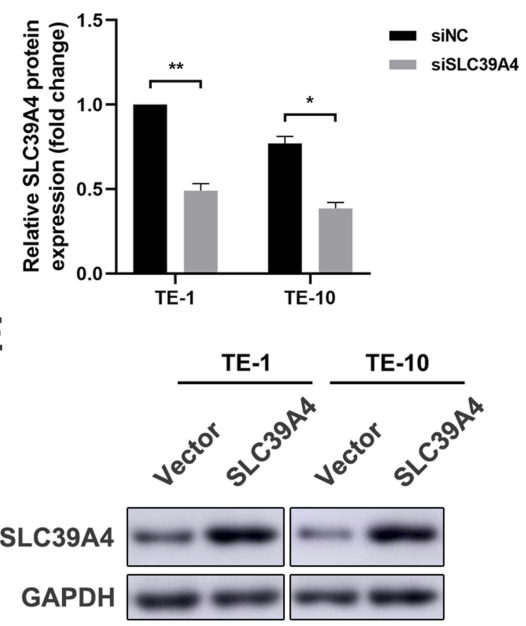

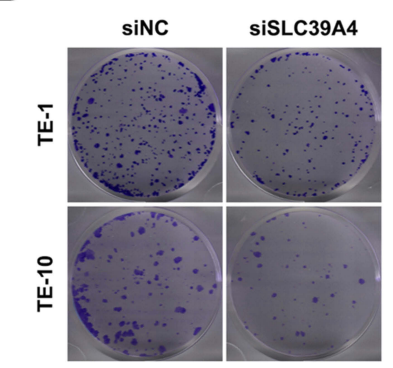

G

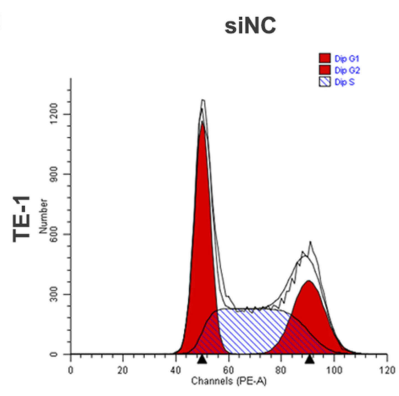

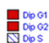

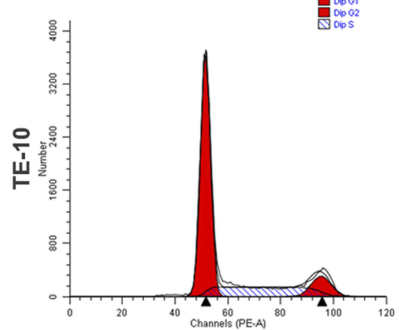

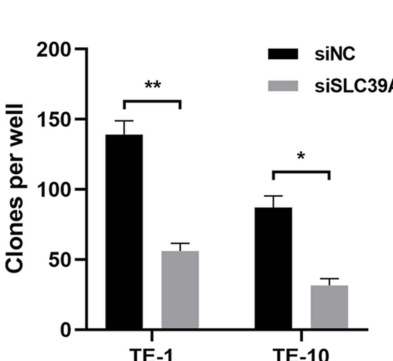

siSLC39A4

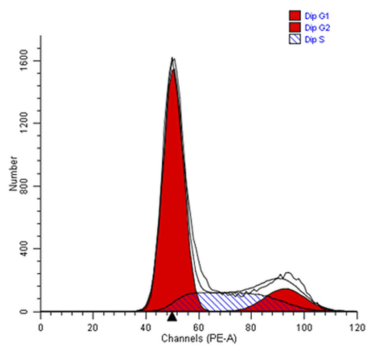

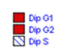

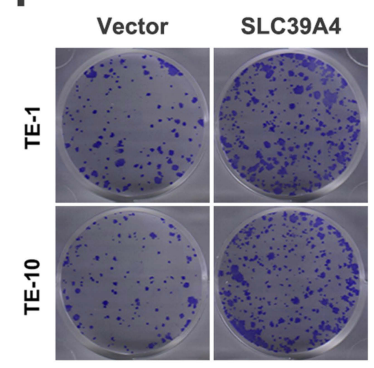

H

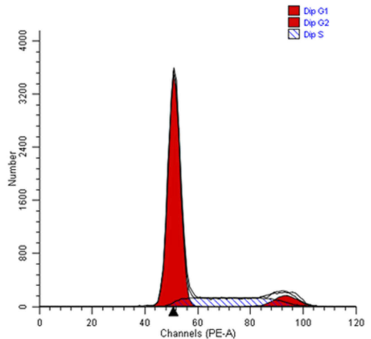

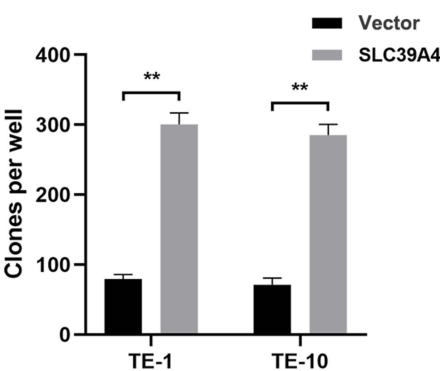

TE-10
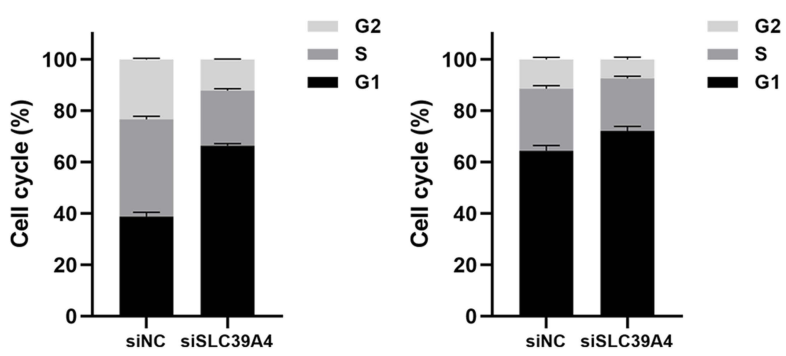

I

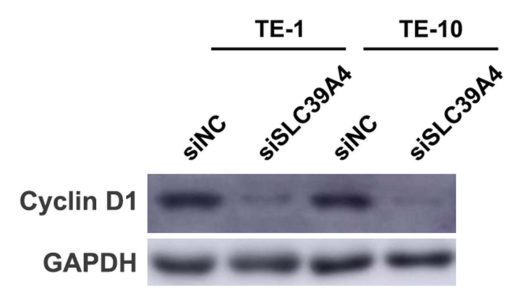

Figure 2 SLC39A4 promotes proliferation in ESCC cells in vitro. (A and B) Knockdown of SLC39A4 expression via a pool of siRNAs in TE-I and TE-I0 cells respectively. The results of qPCR (A) and immunoblotting (B) showed significantly reduced mRNA and protein levels of SLC39A4. GAPDH was used as an internal control. (C) Growth curve suggested that reduced SLC39A4 significantly inhibited cell proliferation in TE-I and TE-I0 cells. (D) Reduction of colony formation capacity in SLC39A4-silenced ESCC cells. (E) The result of immunoblotting showed that SLC39A4 expression was increased in ESCC cells transfected with pEnter-SLC39A4 plasmid. (F) The colony formation capacity was significantly enhanced in both TE-I and TE- 10 cells overexpressing SLC39A4. (G and H) SLC39A4 knockdown-induced G0/G I phase arrest of TE-I and TE-I0 cells $(P<0.05)$. (I) TE-I and TE-I0 cells were transfected with siSLC39A4 for 48 hours, and then the expression level of cell cycle-related protein Cyclin DI was analyzed by immunoblotting. Data in this figure, mean $\pm S D, * P<0.05, * * P<0.01, * * * P<0.001$, $* * * * P<0.0001$.

another vital cause of poor prognosis in ESCC. Because a platinum-based regimen is the standard chemotherapy for ESCC, we explored whether SLC39A4 could affect the sensitivity of ESCC cells to CDDP. As shown in Figure 4A, the apoptosis rate of ESCC cells pretransfected with SLC39A4 siRNAs was significantly 
A

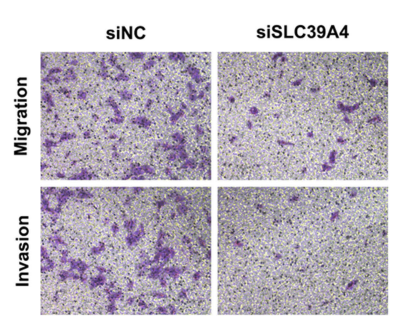

siNC

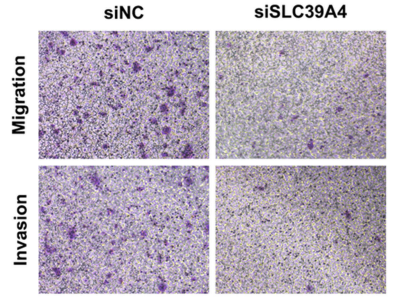

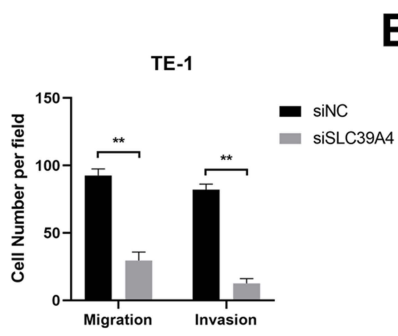

B
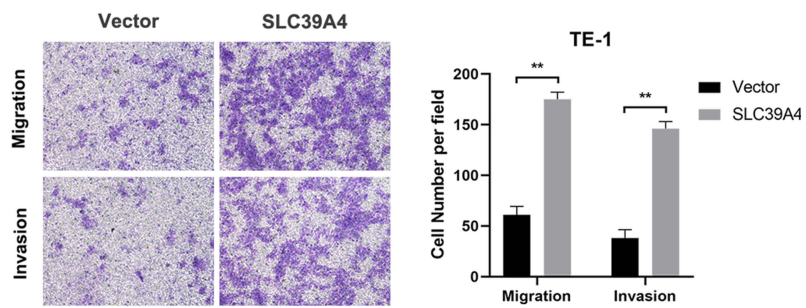

C
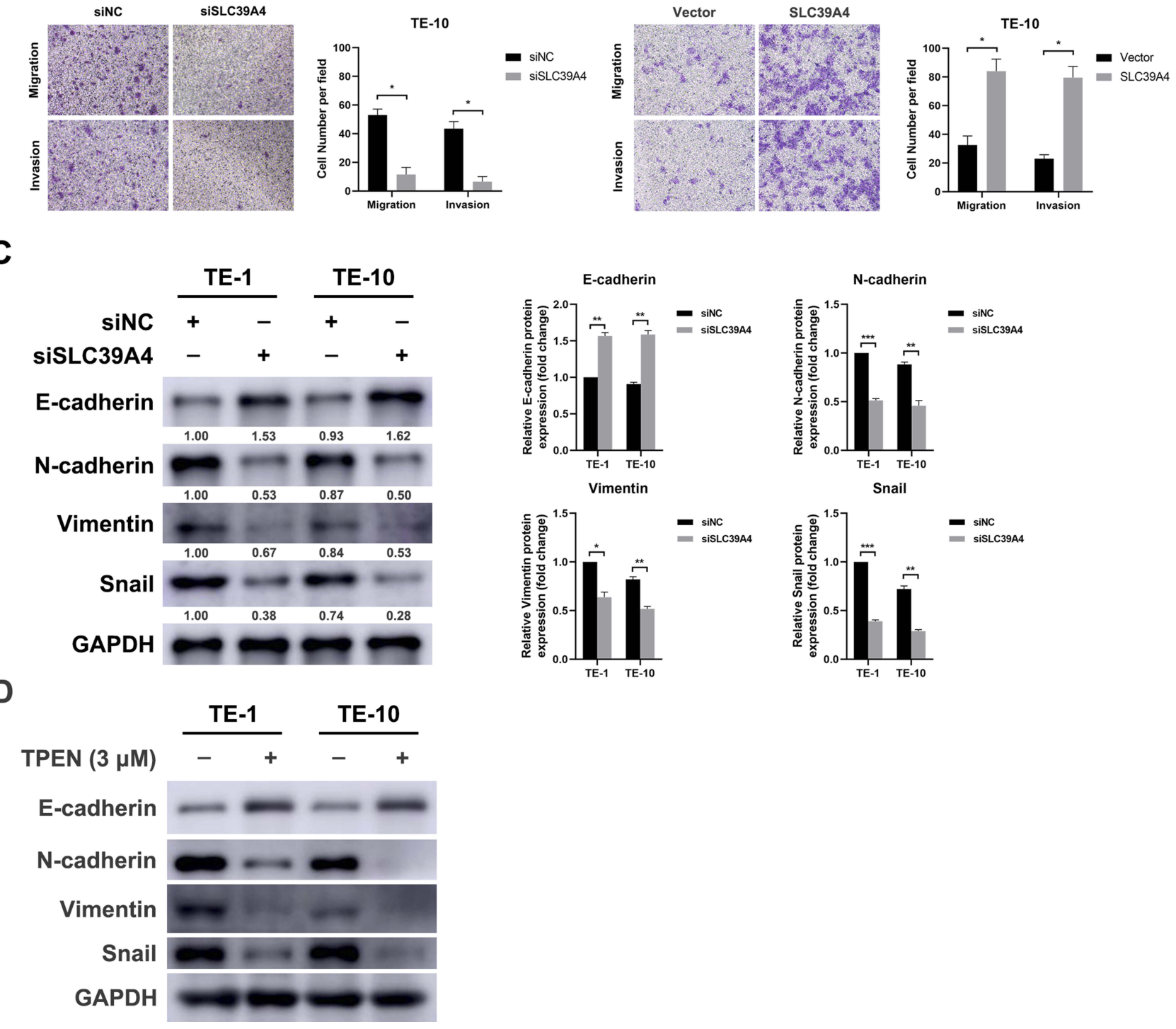

Figure 3 SLC39A4 enhances motility of ESCC cells in vitro. (A) Transwell assay showed that depletion of SLC39A4 dramatically attenuated the abilities of migration and invasion of TE-I and TE- 10 cells. Magnification, I00x. Data, mean \pm SD, $* P<0.05$, $* * P<0.01$. (B) TE-I and TE- 10 cells were transfected with $p$ Enter-SLC39A4 for 48 hours, and the migration and invasion capacities of TE-I and TE-I0 cells were measured by using transwell assay. Magnification, I00x. Data, mean \pm SD, $* P<0.05, * * P<0.01$. (C) The results of immunoblotting analysis showed that depletion of SLC39A4 significantly elevated E-cadherin expression, while the levels of N-cadherin, Vimentin and Snail were significantly reduced. Data, mean $\pm S D$, $* P<0.05$, $* * P<0.01$, $* * * P<0.001$. (D) TE-I and TE-I0 cells were treated with TPEN $(3 \mu M)$ for 24 hours, and the expression of EMT-related proteins was analyzed by immunoblotting. GAPDH was used as an internal control.

increased after exposure to CDDP for 24 hours. Inversely, over-expression of SLC39A4 inhibited CDDP-mediated apoptosis (Figure 4B). Furthermore, SLC39A4 silencing and TPEN treatment both remarkably decreased the $\mathrm{IC}_{50}$ to CDDP in TE-1 and TE-10 cells (Figure 4C and D), suggesting the cells became more sensitive to drugs. These results indicate that SLC39A4 weakened CDDPmediated apoptosis of ESCC cells in vitro. 


\section{Discussion}

$\mathrm{Zn}^{2+}$ is an essential trace element which plays a predominant role in maintaining the activities of various enzymes and transcription factors that contain zinc finger motifs. ${ }^{23,24}$ Recent studies demonstrate that zinc availability is critical for tumor progression because many enzymes involved in hypoxia, angiogenesis, cell proliferation and metastasis function in a zinc-dependent manner. ${ }^{25,26}$ As one crucial zinc transporter, SLC39A4 promotes extracellular zinc uptake and vesicular zinc release into the cytoplasm to increase intracellular zinc availability. ${ }^{27}$ The zinc concentration in tumor cells is much higher than that in normal cells, which may be partially caused by the aberrant expression of SLC39A4. Previous studies show that SLC39A4 is up-regulated significantly in pancreatic cancer, $^{18}$ ovarian cancer $^{28}$ and oral squamous cell

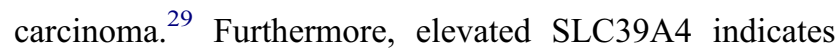
unfavorable prognosis in nasopharyngeal carcinoma ${ }^{30}$ and glioma, $^{22}$ respectively. However, the level of SLC39A4 is significantly down-regulated in prostate carcinoma tissues relative to that in benign prostatic hyperplasia (BPH) tissues. ${ }^{31}$ Consequently, the expression of SLC39A4 may be dependent on the context of specific tumor. In esophageal squamous cell carcinoma (ESCC), we found that the mRNA expression of SLC39A4 was significantly enhanced, which re-confirmed the analysis
A

TE-1

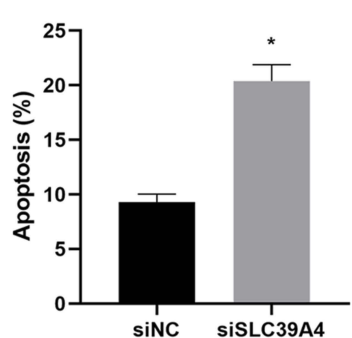

C

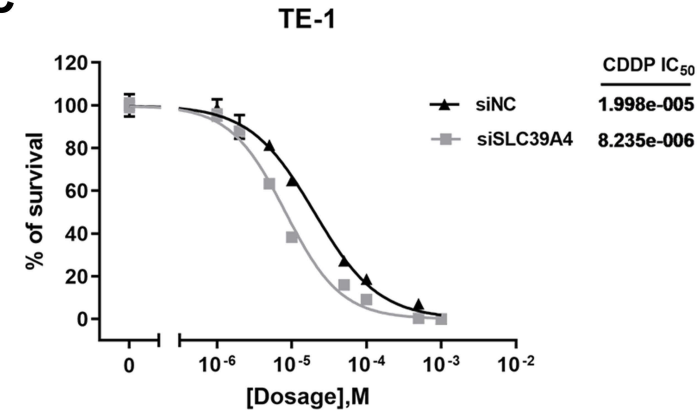

D

TE-1

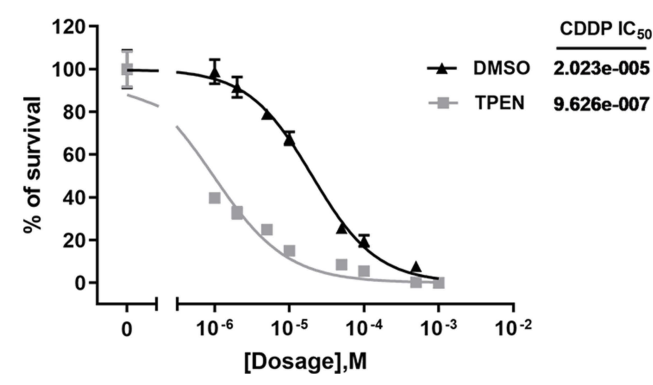

B

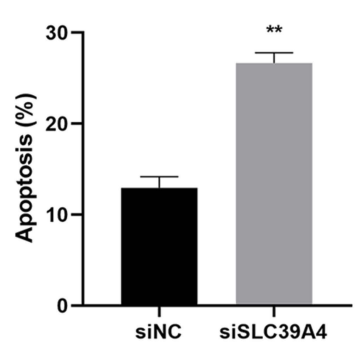

TE-1

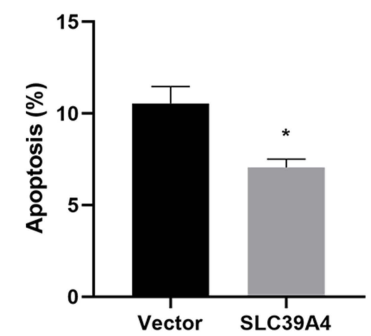

TE-10

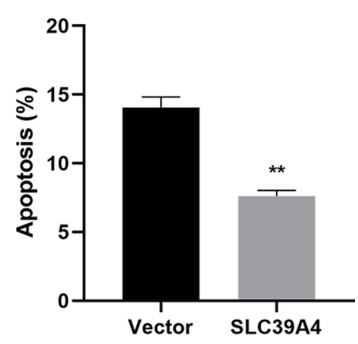

TE-10

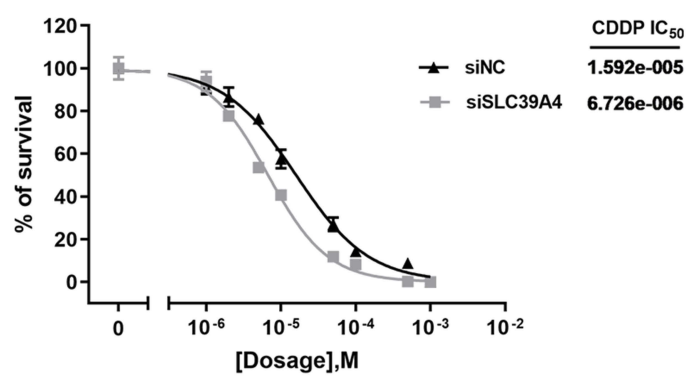

TE-10

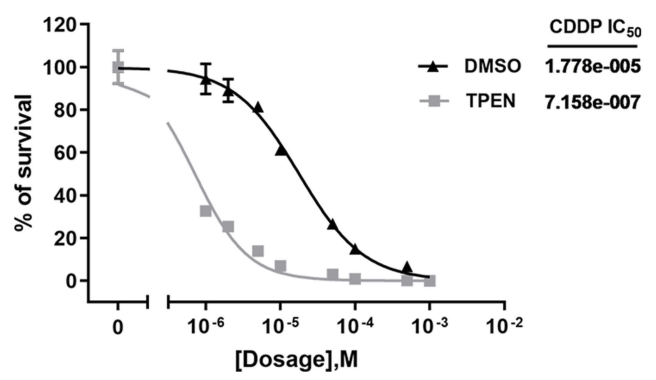

Figure 4 SLC39A4 strengthens chemoresistance of ESCC cells in vitro. (A) SLC39A4-depleted TE-I or TE-10 cells underwent increased apoptosis compared to the negative control. Data, mean \pm SD, $* P<0.05$, $* * P<0.01$. (B) Over-expression of SLC39A4 reduced cisplatin-induced apoptosis in TE-I and TE- I0 cells. Data, mean \pm SD, $* P<0.05$, $* * P<0.01$. (C) Until transfected with siNC or siSLC39A4 for 48 hours, ESCC cells were treated with cisplatin in different dosages for 24 hours. The results of cisplatin IC50 detection showed that depletion of SLC39A4 circumvented the resistance toward cisplatin in TE-I and TE-I0 cells. Bar, SD for quintuplicate wells. (D) The reduced $I_{50}$ to cisplatin was observed in both TE-I and TE-I0 cells treated with TPEN $(3 \mu \mathrm{M})$. Bar, SD for quintuplicate wells. CDDP, cisplatin. IC50, the half maximal inhibitory concentration. 
results of TCGA and GEO databases. The correlation analysis revealed that SLC39A4 was positively correlated with clinicopathological features of ESCC, such as clinical stage, T categories and lymph node metastasis. Moreover, higher expression of SLC39A4 indicated shorter overall survival of ESCC patient. Therefore, SLC39A4 could serve as a prognostic marker in ESCC and may function as an oncogene to promote ESCC progression.

After uncovering the clinical significance of SLC39A4 in ESCC, we continued to explore its role in ESCC progression. Zinc is necessary for growth, while zinc deficiency results in growth retardation. ${ }^{32}$ Previous studies pinpoint that SLC39A4 could promote pancreatic cancer cell growth by activating CREB-IL/STAT3-Cyclin D1 pathway. ${ }^{15,33}$ Therefore, we supposed whether SLC39A4 could affect the growth of ESCC cells. The results of CCK-8 assay and clonogenicity assay demonstrated that knockdown of SLC39A4 by specific siRNAs significantly impaired ESCC cells growth, probably caused by siSLC39A4-induced downregulation of Cyclin D1 and cell cycle arrest.

In view of the positive correlation between SLC39A4 expression and lymph node metastasis in ESCC, the effect of SLC39A4 on ESCC cell motility was detected. The migration and invasion capacities of SLC39A4-deficient ESCC cells were significantly attenuated compared to the control cells, which was in accordance with the previous reports. ${ }^{17,30}$ And we speculated that SLC39A4 may facilitate local invasion and distant metastasis of ESCC cells, which should be further verified by in vivo experiments in the future. As a critical contributor to tumor metastasis, epithelial-mesenchymal transition (EMT) is a process by which epithelial cells lose cell polarity and intercellular adhesion, and obtain migratory and invasive properties to become mesenchymal-like cells. ${ }^{34}$ In this study, we found that SLC39A4 blockage increased the expression of the epithelial marker E-cadherin, but reduced the level of mesenchymal markers, including N-cadherin and Vimentin. Besides, Snail, a critical transcription factor of EMT, was down-regulated in SLC39A4-deficient ESCC cells, revealing that SLC39A4 knockdown reversed EMT process. These data suggested that SLC39A4 may promote ESCC metastasis by triggering EMT.

In addition to distant metastasis, drug resistance is another main cause of poor prognosis in ESCC. In nasopharyngeal carcinoma, inhibition of SLC39A4 enhances the tumor cell sensitivity to radiotherapy, ${ }^{30}$ the main therapeutic strategy for nasopharyngeal carcinoma. Here, we confirmed that siSLC39A4 could significantly sensitize ESCC cells to CDDP in vitro, the standard chemotherapy regimen for ESCC, suggesting SLC39A4 knockdown may increase the response rate and decrease the side effect caused by chemotherapy. Recent studies show that EMT contributes to chemoresistance, ${ }^{35,36}$ therefore, we hold that SLC39A4 may regulate CDDP sensitivity of ESCC cells by modulating EMT process.

\section{Conclusion}

Collectively, we found that SLC39A4 expression was increased in ESCC tissues and this elevation predicted the unfavorable prognosis of patients. Moreover, the expression of SLC39A4 was positively correlated with clinical stage, $\mathrm{T}$ categories and lymph node metastasis in ESCC. Suppression of SLC39A4 expression in ESCC cells undermined the cellular viability, motility capacity and resistance to chemotherapy. These functions of SLC39A4 were at least partially mediated by its implication into epithelial-mesenchymal transition. Although more details of SLC39A4 in ESCC progression remain unclear, we based on these data believe that this zinc transporter could be a promising target in future clinical practice of ESCC.

\section{Disclosure}

The authors report no conflicts of interest in this work.

\section{References}

1. Kamangar F, Dores GM, Anderson WF. Patterns of cancer incidence, mortality, and prevalence across five continents: defining priorities to reduce cancer disparities in different geographic regions of the world. J Clin Oncol. 2006;24(14):2137-2150. doi:10.1200/ JCO.2005.05.2308

2. Rustgi AK, El-Serag HB, Ingelfinger JR. Esophageal carcinoma. $N$ Engl J Med. 2014;371(26):2499-2509. doi:10.1056/NEJMr a1314530

3. Pennathur A, Gibson MK, Jobe BA, et al. Oesophageal carcinoma. Lancet. 2013;381(9864):400-412. doi:10.1016/S0140-6736(12) 60643-6

4. Sun R, Ye L, Zhang M, et al. Prognostic significance of interferon regulating factor 4 in esophageal squamous cell carcinoma. Biochem Biophys Res Commun. 2018;506(3):685-691. doi:10.1016/j. bbrc.2018.10.142

5. Lin L, Yee SW, Kim RB, et al. SLC transporters as therapeutic targets: emerging opportunities. Nat Rev Drug Discov. 2015;14(8):543-560. doi: $10.1038 /$ nrd4626

6. Bai X, Moraes TF, Reithmeier RAF. Structural biology of solute carrier (SLC) membrane transport proteins. Mol Membr Biol. 2017;34(1-2):1-32. doi:10.1080/09687688.2018.1448123

7. Hediger MA, Romero MF, Peng JB, et al. The ABCs of solute carriers: physiological, pathological and therapeutic implications of human membrane transport proteinsIntroduction. Pflugers Arch. 2004;447 (5):465-468. doi:10.1007/s00424-003-1192-y 
8. Schlessinger A, Khuri N, Giacomini KM, et al. Molecular modeling and ligand docking for solute carrier (SLC) transporters. Curr Top Med Chem. 2013;13(7):843-856. doi:10.2174/1568026611 313070007

9. Hara T, Takeda TA, Takagishi T, et al. Physiological roles of zinc transporters: molecular and genetic importance in zinc homeostasis. J Physiol Sci. 2017;67(2):283-301. doi:10.1007/s12576-017-0521-4

10. Bhutia YD, Babu E, Ramachandran S, et al. SLC transporters as a novel class of tumour suppressors: identity, function and molecular mechanisms. Biochem J. 2016;473(9):1113-1124. doi:10.1042/ BJ20150751

11. Thorsen K, Mansilla F, Schepeler T, et al. Alternative splicing of SLC39A14 in colorectal cancer is regulated by the Wnt pathway. Mol Cell Proteomics. 2011;10(1):M110 002998. doi:10.1074/mcp. M110.002998

12. Cui XB, Shen YY, Jin TT, et al. SLC39A6: a potential target for diagnosis and therapy of esophageal carcinoma. $J$ Transl Med. 2015;13(1):321. doi:10.1186/s12967-015-0681-z

13. Sheng N, Yan L, You W, et al. Knockdown of SLC39A7 inhibits cell growth and induces apoptosis in human colorectal cancer cells. Acta Biochim Biophys Sin (Shanghai). 2017;49(10):926-934. doi:10.1093/ abbs/gmx094

14. Kagara N, Tanaka N, Noguchi S, et al. Zinc and its transporter ZIP10 are involved in invasive behavior of breast cancer cells. Cancer Sci. 2007;98(5):692-697. doi:10.1111/j.1349-7006.2007.00446.x

15. Li M, Zhang Y, Bharadwaj U, et al. Down-regulation of ZIP4 by RNA interference inhibits pancreatic cancer growth and increases the survival of nude mice with pancreatic cancer xenografts. Clin Cancer Res. 2009;15(19):5993-6001. doi:10.1158/1078-0432.CCR-09-0557

16. Zhang Y, Yang J, Cui X, et al. A novel epigenetic CREB-miR-373 axis mediates ZIP4-induced pancreatic cancer growth. EMBO Mol Med. 2013;5(9):1322-1334. doi:10.1002/emmm.201302507

17. Liu M, Yang J, Zhang Y, et al. ZIP4 promotes pancreatic cancer progression by repressing ZO-1 and claudin-1 through a ZEB1-dependent transcriptional mechanism. Clin Cancer Res. 2018;24(13):3186-3196. doi:10.1158/1078-0432.CCR-18-0263

18. Li M, Zhang Y, Liu Z, et al. Aberrant expression of zinc transporter ZIP4 (SLC39A4) significantly contributes to human pancreatic cancer pathogenesis and progression. Proc Natl Acad Sci U S A. 2007;104(47):18636-18641. doi:10.1073/pnas.0709307104

19. Cui X, Zhang Y, Yang J, et al. ZIP4 confers resistance to zinc deficiency-induced apoptosis in pancreatic cancer. Cell Cycle. 2014;13(7):1180-1186. doi:10.4161/cc.28111

20. Yang J, Zhang Z, Zhang Y, et al. ZIP4 promotes muscle wasting and cachexia in mice with Orthotopic pancreatic tumors by stimulating RAB27B-regulated release of extracellular vesicles from cancer cells. Gastroenterology. 2019;156(3):722-734 e6. doi:10.1053/j. gastro.2018.10.026

21. Wu DM, Liu T, Deng SH, et al. SLC39A4 expression is associated with enhanced cell migration, cisplatin resistance, and poor survival in non-small cell lung cancer. Sci Rep. 2017;7(1):7211. doi:10.1038/ s41598-017-07830-4
22. Lin Y, Chen Y, Wang Y, et al. ZIP4 is a novel molecular marker for glioma. Neuro Oncol. 2013;15(8):1008-1016. doi:10.1093/neuonc/ not042

23. Mao X, Kim BE, Wang F, et al. A histidine-rich cluster mediates the ubiquitination and degradation of the human zinc transporter, hZIP4, and protects against zinc cytotoxicity. J Biol Chem. 2007;282 (10):6992-7000. doi:10.1074/jbc.M610552200

24. Wang K, Zhou B, Kuo YM, et al. A novel member of a zinc transporter family is defective in acrodermatitis enteropathica. $\mathrm{Am}$ J Hum Genet. 2002;71(1):66-73. doi:10.1086/341125

25. Juhasz M, Chen J, Lendeckel U, et al. Expression of carbonic anhydrase IX in human pancreatic cancer. Aliment Pharmacol Ther. 2003;18(8):837-846. doi:10.1046/j.1365-2036.2003.01738.x

26. Garcea G, Doucas H, Steward WP, et al. Hypoxia and angiogenesis in pancreatic cancer. ANZ J Surg. 2006;76(9):830-842. doi:10.1111/ j.1445-2197.2006.03872.x

27. Cousins RJ, Liuzzi JP, Lichten LA. Mammalian zinc transport, trafficking, and signals. J Biol Chem. 2006;281(34):24085-24089. doi:10.1074/jbc.R600011200

28. Fan Q, Cai Q, Li P, et al. The novel ZIP4 regulation and its role in ovarian cancer. Oncotarget. 2017;8(52):90090-90107. doi:10.18632/ oncotarget. 21435

29. Ishida S, Kasamatsu A, Endo-Sakamoto Y, et al. Novel mechanism of aberrant ZIP4 expression with zinc supplementation in oral tumorigenesis. Biochem Biophys Res Commun. 2017;483 (1):339-345. doi:10.1016/j.bbrc.2016.12.142

30. Zeng Q, Liu YM, Liu J, et al. Inhibition of ZIP4 reverses epithelial-to-mesenchymal transition and enhances the radiosensitivity in human nasopharyngeal carcinoma cells. Cell Death Dis. 2019;10(8):588. doi:10.1038/s41419-019-1807-7

31. Chen QG, Zhang Z, Yang Q, et al. The role of zinc transporter ZIP4 in prostate carcinoma. Urol Oncol. 2012;30(6):906-911. doi:10.1016/ j.urolonc.2010.11.010

32. Takeda A, Goto K, Okada S. Zinc depletion suppresses tumor growth in mice. Biol Trace Elem Res. 1997;59(1-3):23-29. doi:10.1007/ BF02783226

33. Zhang Y, Bharadwaj U, Logsdon CD, et al. ZIP4 regulates pancreatic cancer cell growth by activating IL-6/STAT3 pathway through zinc finger transcription factor CREB. Clin Cancer Res. 2010;16 (5):1423-1430. doi:10.1158/1078-0432.CCR-09-2405

34. Chaffer CL, San Juan BP, Lim E, et al. EMT, cell plasticity and metastasis. Cancer Metastasis Rev. 2016;35(4):645-654. doi:10.1007/s10555-016-9648-7

35. Fischer KR, Durrans A, Lee S, et al. Epithelial-to-mesenchymal transition is not required for lung metastasis but contributes to chemoresistance. Nature. 2015;527(7579):472-476. doi:10.1038/ nature 15748

36. Zheng X, Carstens JL, Kim J, et al. Epithelial-to-mesenchymal transition is dispensable for metastasis but induces chemoresistance in pancreatic cancer. Nature. 2015;527(7579):525-530. doi:10.1038/ nature 16064
OncoTargets and Therapy

\section{Publish your work in this journal}

OncoTargets and Therapy is an international, peer-reviewed, open access journal focusing on the pathological basis of all cancers, potential targets for therapy and treatment protocols employed to improve the management of cancer patients. The journal also focuses on the impact of management programs and new therapeutic agents and protocols on patient perspectives such as quality of life, adherence and satisfaction. The manuscript management system is completely online and includes a very quick and fair peer-review system, which is all easy to use. Visit http://www.dovepress.com/ testimonials.php to read real quotes from published authors. 